\section{(6) OPEN ACCESS}

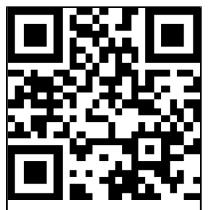

Open Access Scan to access mo free content

- Additional material is published online only. To view please visit the journal online (http://dx.doi.org/10.1136/ gutjnl-2012-303207)

${ }^{1}$ Department of Medical Biochemistry, University of Gothenburg, Gothenburg, Sweden

${ }^{2}$ Cardiovascular Biology Research Program, Oklahoma Medical Research Foundation, Oklahoma City, Oklahoma, USA

${ }^{3}$ Department of Pediatrics, University of Arizona Health Sciences Center, Tucson, Arizona, USA

${ }^{4}$ Department of Biology, Georgia State University, Atlanta, Georgia, USA

${ }^{5}$ Department Internal Medicine University of Gothenburg, Gothenburg, Sweden

\section{Correspondence to} Dr Gunnar C Hansson, Department of Medical Biochemistry, University of Gothenburg, Box 440, Gothenburg 405 30, Sweden; gunnar.hansson@medkem.gu. se

Received 29 June 2012 Accepted 28 January 2013 Published Online First 20 February 2013

\section{SLinked}

- http://dx.doi.org/10.1136/ gutjnl-2012-304602.pdf

To cite: Johansson MEV, Gustafsson JK, HolménLarsson J, et al. Gut 2014:63:281-291.

\title{
Bacteria penetrate the normally impenetrable inner colon mucus layer in both murine colitis models and patients with ulcerative colitis
}

\author{
Malin E V Johansson, ${ }^{1}$ Jenny K Gustafsson, ${ }^{1}$ Jessica Holmén-Larsson, ${ }^{1}$ \\ Karolina S Jabbar, ${ }^{1}$ Lijun Xia, ${ }^{2}$ Hua Xu, ${ }^{3}$ Fayez K Ghishan, ${ }^{3}$ Frederic A Carvalho, ${ }^{4}$ \\ Andrew T Gewirtz, ${ }^{4}$ Henrik Sjövall, ${ }^{5}$ Gunnar C Hansson ${ }^{1}$
}

\section{ABSTRACT}

Objective The inner mucus layer in mouse colon normally separates bacteria from the epithelium. Do humans have a similar inner mucus layer and are defects in this mucus layer a common denominator for spontaneous colitis in mice models and ulcerative colitis (UC)?

Methods and results The colon mucus layer from mice deficient in Muc2 mucin, Core 10 -glycans, Tlr5, interleukin 10 (IL-10) and Slc9a3 (Nhe3) together with that from dextran sodium sulfate-treated mice was immunostained for Muc2, and bacterial localisation in the mucus was analysed. All murine colitis models revealed bacteria in contact with the epithelium. Additional analysis of the less inflamed IL-10-1- mice revealed a thicker mucus layer than wild-type, but the properties were different, as the inner mucus layer could be penetrated both by bacteria in vivo and by fluorescent beads the size of bacteria ex vivo. Clear separation between bacteria or fluorescent beads and the epithelium mediated by the inner mucus layer was also evident in normal human sigmoid colon biopsy samples. In contrast, mucus on colon biopsy specimens from patients with UC with acute inflammation was highly penetrable. Most patients with UC in remission had an impenetrable mucus layer similar to that of controls.

Conclusions Normal human sigmoid colon has an inner mucus layer that is impenetrable to bacteria. The colon mucus in animal models that spontaneously develop colitis and in patients with active UC allows bacteria to penetrate and reach the epithelium. Thus colon mucus properties can be modulated, and this suggests a novel model of UC pathophysiology.

\section{INTRODUCTION}

Inflammatory bowel disease, which is on the rise in the Western world, can be divided into Crohn's disease (CD) and ulcerative colitis (UC). Both these diseases are dependent on the presence of intestinal microbiota, although specific bacteria have not been linked to disease. ${ }^{1}{ }^{2}$ Classical CD has been genetically and functionally linked to proteins such as NOD2 and ATG16L1. ${ }^{3}$ UC is not as well understood as CD, is restricted to the colon and rectum, and is characterised by superficial mucosal inflammation. ${ }^{4}$ The disease is likely to occur in genetically predisposed individuals in combination with

\section{Significance of this study}

What is already known about this subject?

- In mouse colon, bacteria are separated from the epithelial cells by the inner mucus layer formed by MUC2 mucin.

- Muc2-deficient mice do not have any secreted mucus and have bacteria in direct contact with the epithelium. These mice develop colitis and later on colon cancer.

What are the new findings?

- Humans have an inner mucus layer that normally separates bacteria from coming in contact with the epithelial cells.

- Penetration of the inner mucus layer by beads the size of bacteria correlate with bacterial penetration.

- Mouse models of colitis have bacteria and beads penetrating the inner mucus layer.

- Humans with active ulcerative colitis (UC) have an inner mucus layer that is penetrable.

- Some patients with UC in remission also have an inner mucus layer that is penetrable.

How might it impact on clinical practice in the foreseeable future?

- This observation will give a new understanding of UC and open new lines of research.

- An understanding of how the penetrability properties of the inner mucus are regulated will provide novel therapeutic opportunities. Improved mucus protection could be instrumental for maintenance of UC remission.

environmental and microbial exposure. ${ }^{5}$ A morphological characteristic used to assess disease activity is the typical goblet cell depletion reflecting emptied goblet cells, something that may suggest a role for the mucus system in UC.

The gut epithelium manages enteric bacterial flora in a way that allows a symbiotic relation between host and microbes. The functions, and thus the needs, of the epithelium are different along the intestine, and protection of these surfaces are accordingly handled in different ways. ${ }^{6}$ The 
small-intestinal epithelium mediates nutritional uptake, and the mucus covering these surfaces allows this transport. The bacterial load of the intestine, however, increases dramatically in the distal direction with massive colonisation in the colon. These numerous bacteria are normally handled without causing inflammation, but sometimes the immune system is strongly activated and severe inflammation is observed. How this balance is controlled is still not fully understood, but the recently discovered inner mucus layer in the colon, which is impervious to bacteria and physically separates bacteria from the epithelium, has provided a novel insight into this function. ${ }^{7}$ It has been suggested that there is also a separation between bacteria and epithelium in humans, ${ }^{2} 89$ but it is still not clear if humans have a similar protective mucus layer formed by MUC2 mucin, although a stratified mucus layer has been suggested. ${ }^{10}$

Colonic mucus is produced by the goblet cell, a cell type devoted to this task. The main mucus component in the intestine is MUC2 mucin, a large and heavily O-glycosylated gelforming mucin that forms enormous polymeric nets by C-terminal dimerisation and $\mathrm{N}$-terminal trimerisation. ${ }^{11}{ }^{12}$ Upon secretion from the goblet cells, the mucus expands rapidly and builds a stratified dense layer that is attached to the epithelium. ${ }^{13}$ This mucus contains additional proteins, of which the FCGBP protein is important as it can stabilise the mucus by additional cross-links. ${ }^{14}$ At a distance, far from the epithelial surface, the inner mucus is transformed into a soluble and less organised outer mucus layer which, by proteolytic expansion, generates the preferred habitat for the commensal microbes. ${ }^{11}$ Development of spontaneous colitis in Muc2-deficient mice confirms the protective properties of the inner colonic mucus layer. ${ }^{715}$ In these mice, bacteria are found in direct contact with the epithelial cells, far down in the colonic crypts and inside enterocytes, locations never observed in wild-type (WT) animals. These observations and earlier studies on humans with UC showing bacteria in contact with the epithelium ${ }^{16}{ }^{17}$ may suggest that defects in the inner mucus layer that allow bacteria to reach the epithelium in larger quantities than normal can activate the immune system. This prompted us to address, both in mouse models and human UC, whether there are defects of the inner colon mucus layer that can be linked to colitis. We now show that colitic mouse models and patients with UC have dysfunctional colonic mucus that can be penetrated by bacteria.

\section{MATERIALS AND METHODS}

\section{Animals}

Experiments were performed using WT C57/Bl6, IL-10 ${ }^{-/-}$, Slc9a3 ${ }^{-/-}$, C1galt $1^{-1-}$ and $\mathrm{Tlr} 5^{-/-}$mice all on a C57/Bl6 background (male 8-12 weeks) according to local ethics committee guidelines. All experiments included controls from the same animal facility as the knock-out strains. Dextran sodium sulfate (DSS) experiments were performed as described. ${ }^{18} 19$

\section{Human subjects and biopsies}

Subjects were recruited from among patients referred for colonoscopy at Sahlgrens' University Hospital, Gothenburg, Sweden. Biopsy specimens from the sigmoid colon of 28 patients with UC were assessed. Disease activity was determined by the Mayo endoscopic score. ${ }^{20}$ Clinical information on the patients with UC is presented in table 1 . Biopsy samples were also obtained from 12 patients with macro- and micro-scopically normal mucosa (control group). Clinical information on these patients is presented in online supplementary table S1. Written and informed consent was obtained from all study subjects, and approval for this study was granted by the Human Research
Ethics Committee, University of Gothenburg. Biopsy samples were collected one at a time using single-use large-capacity forceps (Olympus) and instantly put into ice-cold oxygenated Krebs solution or fixed in Carnoy's fixative. ${ }^{7}$

\section{Fluorescence in situ hybridisation (FISH) and immunostaining}

Paraffin-embedded Carnoy-fixed sections were dewaxed and stained with H\&E or Alcian blue/periodate acid schiff (PAS) or hybridised with $10 \mathrm{ng} / \mu \mathrm{l}$ of a general bacterial $16 \mathrm{~S} \mathrm{rRNA}$ probe (EUB338) and immunostained for Muc2 using the MUC2C3 antiserum or for DNA by 4',6-diamidino-2-phenylindole (DAPI). ${ }^{7}$ Images were obtained with an Axio Examiner Z1 LSM 700 confocal microscope and ZEN 2010 software (Zeiss).

\section{Mucus penetration score, histology score and goblet cell measurements}

Mucus penetration by bacteria was blindly evaluated on DNA-stained sections ( $\mathrm{n}=5$ for IL- $10^{-/}$and WT) by two independent observers. The scores $(0-4)$ were based on observations from a whole intestinal cross-section per sample, and increased scores correspond to increased contact between bacteria and epithelium. Histological scoring of inflammation was performed on H\&E- and Alcian blue/PAS-stained sections ( $n=3$ per genotype) in a blinded fashion by two independent observers evaluating one whole tissue section per sample. The sum of scores for inflammatory cell infiltration (score, 0-4), goblet cell depletion or decreased mucus accumulation (score, 0-4), mucosa thickening (score, 0-4), destruction of architecture (score, 0 or 3-4) and loss of crypts (score, 0 or 3-4) was calculated (maximum 20). All data are presented as mean \pm SEM.

Number of goblet cells was counted as described in the online supplement. Data are presented as mean \pm SEM with 34 human samples (10 controls, 14 patients with UC in remission, 10 patients with active disease) and 10 mouse samples (five WT, five IL- $10^{-/-}$). Mucus-filled theca area of goblet cells was measured on pictures of anti-MUC2C3-stained sections as described in the online supplement. Data are presented as mean area \pm SEM with 34 human samples (10 controls, 14 patients with $\mathrm{UC}$ in remission, 10 patients with active disease) and 10 mouse samples (five WT, five IL-10 $0^{-/}$).

\section{Preparation of mouse tissue for in vivo and ex vivo experiments}

For the in vivo experiments, mice were anaesthetised by continuous administration of isoflurane (Isoba vet; Schering Plough). The abdomen was opened and a cup was placed on the opened intestinal segment and filled with warm saline. For the ex vivo experiments, mice were anaesthetised with isoflurane and killed by cervical dislocation. The distal colon was dissected, flushed, and the longitudinal muscle layer removed. The tissue explant was mounted in a horizontal perfusion chamber.

\section{Mucus thickness}

The thickness of the colonic mucus was measured as described previously. ${ }^{19}$ Briefly, the upper surface of the colonic mucus was visualised by addition of charcoal particles. The mucus thickness was determined by measuring the distance between the epithelial surface and the mucus surface by a micropipette viewed through a stereomicroscope (Leica MZ12).

\section{Mucus penetrability}

Mucus penetrability was measured as described previously. ${ }^{19}$ Briefly, colonic explants were mounted in a perfusion chamber 
Table 1 Clinical information on the analysed patients with UC

\begin{tabular}{|c|c|c|c|c|c|c|c|}
\hline No & Gender & Age & $\begin{array}{l}\text { Mayo Score } \\
\text { (endoscopic 0-3) }\end{array}$ & $\begin{array}{l}\text { Histological } \\
\text { assessment }\end{array}$ & Medication & $\begin{array}{l}\text { Relapses/year } \\
\text { (disease onset)* }\end{array}$ & $\mathrm{PSC}^{* *}$ \\
\hline 1 & $M$ & 47 & 0 & $A, L$ & 5-ASA & $<1$ & $x$ \\
\hline 2 & $M$ & 24 & 0 & NAD & 5-ASA+azathioprine & $<1$ & $x$ \\
\hline 3 & $M$ & 26 & 0 & $A, L, E$ & 5-ASA+azathioprine & $<1$ & \\
\hline 4 & $M$ & 57 & 0 & NAD & 5-ASA & $<1$ & \\
\hline 5 & $M$ & 29 & 0 & $A$ & 5-ASA & $<1$ & \\
\hline 6 & $M$ & 53 & 0 & $A$ & 5-ASA+TNFa inhibitorst & $<1$ & \\
\hline 7 & $\mathrm{~F}$ & 58 & 0 & NAD & 5-ASA & $<1$ & \\
\hline 8 & $M$ & 32 & 0 & NAD & 5-ASA+azathioprine & $\geq 1$ & \\
\hline $9 \ddagger$ & $M$ & 24 & 0 & A & 5-ASA+azathioprine & $<1$ & $x$ \\
\hline 10 & $M$ & 27 & 0 & $A$ & 5-ASA & (Onset 2011) & \\
\hline 11 & $\mathrm{~F}$ & 22 & 0 & $\mathrm{~L}, \mathrm{C}$ & 5-ASA+steroids & (Onset 2011) & \\
\hline 12 & $M$ & 74 & 0 & $A$ & 5-ASA & $<1$ & \\
\hline 13 & $\mathrm{~F}$ & 55 & 0 & $A$ & 5-ASA & $<1$ & \\
\hline 14 & $M$ & 34 & 0 & NAD & None & $<1$ & \\
\hline 15 & $M$ & 27 & 0 & NAD & 5-ASA & $\geq 1$ & \\
\hline 16 & M & 72 & 0 & NAD & 5-ASA & $<1$ & \\
\hline 17 & $M$ & 38 & 0 & NAD & 5-ASA & (Onset 2011) & \\
\hline 18 & $\mathrm{~F}$ & 65 & 1 & $A, L, E, P, N, C, C A$ & 5-ASA & $<1$ & \\
\hline 19 & $\mathrm{~F}$ & 37 & 1 & $\mathrm{~L}, \mathrm{E}, \mathrm{C}, \mathrm{CA}$ & 5-ASA & $<1$ & \\
\hline 20 & $M$ & 52 & 2 & $L, E, P, C A, U$ & 5-ASA & (Onset 2008) & \\
\hline 21 & $M$ & 28 & 2 & $A, L, E, C, C A$ & 5-ASA & (Onset 2009) & \\
\hline 22 & $\mathrm{~F}$ & 31 & 2 & $A, L, E, C$ & 5-ASA & $\geq 1$ & \\
\hline 23 & $M$ & 36 & 3 & $A, L, N, D$ & 5-ASA+azathioprine & $\geq 1$ & \\
\hline 24 & $M$ & 62 & 1 & $A, L, C$ & 5-ASA+steroids & $\geq 1$ & \\
\hline 25 & $M$ & 26 & 2 & $A, L, E, P, C, C A$ & 5-ASA+azathioprine & $\geq 1$ & \\
\hline 26 & $M$ & 45 & 2 & $A, L, C$ & 5-ASA+azathioprine & $\geq 1$ & \\
\hline 27 & $M$ & 18 & 2 & $A, L, E, P, C, C A, D, U$ & 5-ASA+azathioprine & $\geq 1$ & \\
\hline 28 & $\mathrm{~F}$ & 21 & 1 & $A, L, E, P$ & 5-ASA+TNF $\alpha$ inhibitors & $\geq 1$ & \\
\hline
\end{tabular}

${ }^{*}$ For patients with recent disease onset, the number of relapses cannot be given. Instead year of debut is stated in parentheses.

**PSC, primary sclerosing cholangitis. $x$, means present.

tMedication with TNF $\alpha$ inhibitor for rheumatoid arthritis.

‡This patient had inflammation in more proximal part.

5-ASA, 5-aminosalicylic acid; A, alterations in crypt architecture; C, cryptitis; CA, crypt abscesses; D, denuded epithelium; E, eosinophils; F, female; L, lymphocytes; M, male;

$\mathrm{N}$, neutrophils; NAD, nothing abnormal detected; P, plasma cells; PSC, primary sclerosing cholangitis; TNF, tumour necrosis factor; U, ulceration; UC, ulcerative colitis.

and incubated for $20 \mathrm{~min}$; a suspension of $2 \mu \mathrm{m}$ green beads and $0.5 \mu \mathrm{m}$ red beads was then added to the apical surface (Fluospheres; Invitrogen). The beads were left to sediment through the mucus for $40 \mathrm{~min}$, after which the position of the beads in relation to the epithelium was analysed by taking confocal images in XY stacks with an LSM 700 Axio Examiner Z.1 confocal imaging system and ZEN 2009 software (Zeiss). Results were analysed using Volocity 5.5.1 software (Perkin-Elmer). Mucus penetrability was analysed to reveal beads close to the epithelium and the mucus thickness containing beads. A detailed description of the methods is available in the online supplement.

\section{Statistical analysis}

Mouse data were analysed using a two-tailed Mann-Whitney U test. For the human data, the Kruskal-Wallis test with Dunns' correction for multiple comparisons was used. A p value $<0.05$ was regarded as significant.

Detailed descriptions of the materials and methods can be found in the online supplement.

\section{RESULTS}

The inner mucus layer is defective in animal models that spontaneously develop colitis

The inner mucus layer of the distal colon is formed from sheets of Muc2 mucin organised into stacked layers in a stratified way that does not allow bacteria to penetrate. This physically separates bacteria from the epithelium (figure 1, WT). Animals deficient in the Muc2 gene are devoid of secreted mucus layers, resulting in constant contact between bacteria and the epithelium (figure $1, \mathrm{Muc}^{-/}$). ${ }^{7}$ The presence of bacteria in contact with the epithelium was estimated (figure 2A and online supplementary figure S1A), and the level of inflammation was estimated on H\&E- and Alcian blue/PAS-stained tissue sections evaluating immune cell infiltration, crypt architecture and goblet cell filling ('depletion') (figure 2B,C and online supplementary figure S1B). As observed previously, the $\mathrm{Muc}^{-/-}$animals showed severe inflammation marked by infiltration of leukocytes and increased cell proliferation as evidenced by crypt elongation (figure $2 \mathrm{C}, \mathrm{Muc}^{-/-}$). ${ }^{15}$ A more subtle way to modify Muc2 mucin is to alter its glycosylation by shortening the $\mathrm{O}$-glycans. Blocking the core 1 extensions by deletion of the C1galt 1 glycosyltransferase results in reduced mucin glycosylation, which causes spontaneous colitis. ${ }^{21}$ These animals show a defective inner mucus layer that allows bacteria to penetrate and come in contact with the epithelium (figure 1 and online supplementary figure S1A, C1 galt $1^{-/-}$).

Sodium-hydrogen exchanger Slc9a3 (Nhe3)-deleted mice have been shown to develop spontaneous colitis. ${ }^{22}{ }^{23}$ When colon tissue sections from these mice were analysed, essentially normal mucus was observed with regard to thickness and 

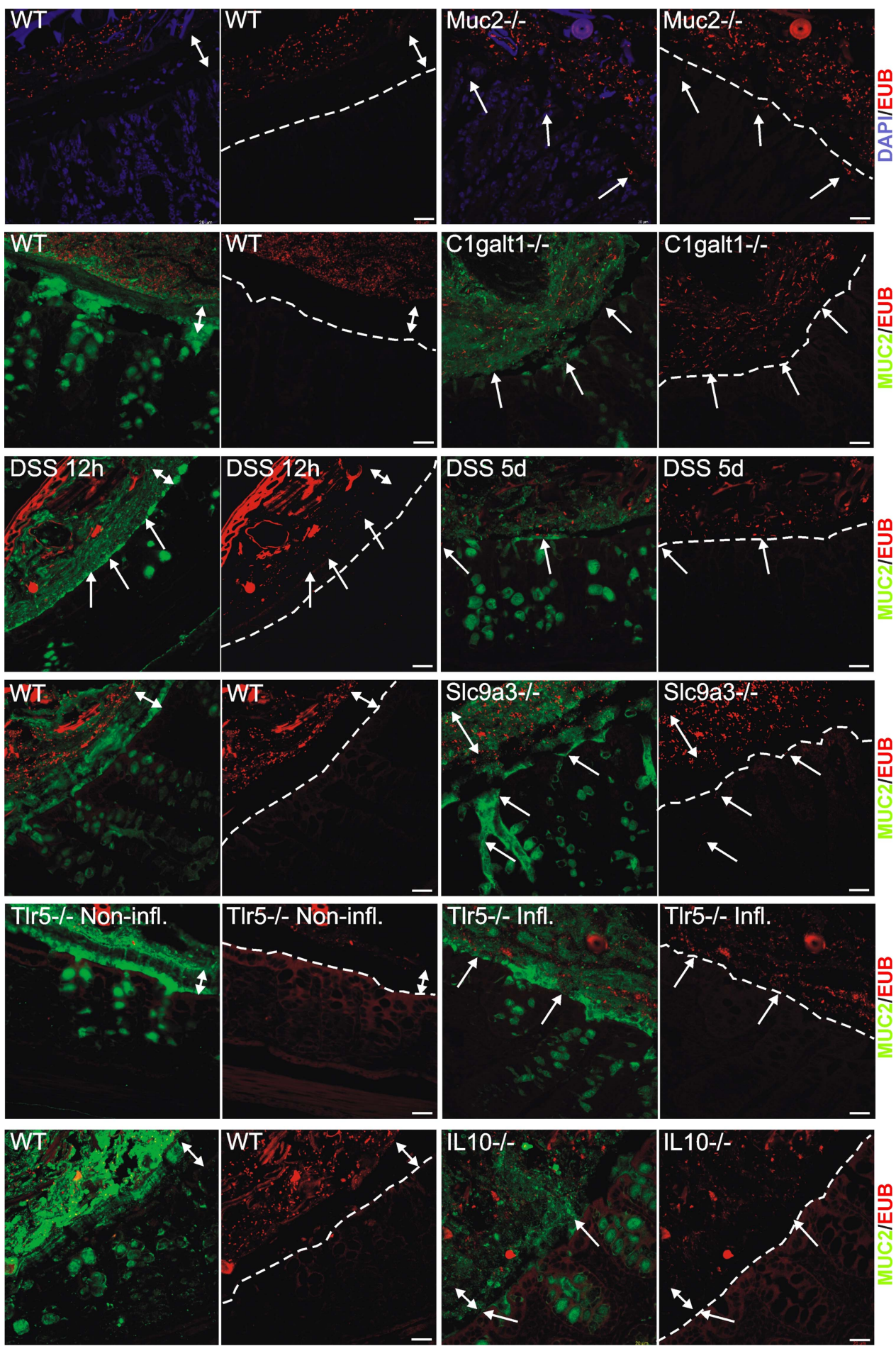

Figure 1 Localisation of bacteria in the inner mucus layer of colon in different mouse colitis models. Fixed colon sections with preserved mucus were immunostained for Muc2 (green) and bacteria as detected by fluorescence in situ hybridisation with general bacterial $16 \mathrm{~S}$ probes (red) and DNA stained using 4',6-diamidino-2-phenylindole (DAPI; blue). Wild-type (WT) was compared with the spontaneous colitis models with different

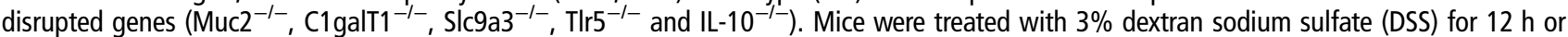
5 days. Doubled-headed arrows show the inner mucus layer not always free of bacteria. Arrows point to bacteria close to the epithelial cells. Scale bars are $20 \mu \mathrm{m}$. Infl., inflamed; Non-infl., non-inflamed.

stratified appearance, but it was penetrated by bacteria (figure 1 and online supplementary figure $\mathrm{S} 1 \mathrm{~A}, \mathrm{Slc} 9 \mathrm{a}^{-/-}$). As previously described, the tissue sections also showed signs of inflammation with infiltration of immune cells (figure 2C and online supplementary figure $\left.\mathrm{S} 1 \mathrm{~B}, \mathrm{Slc} 9 \mathrm{a} 3^{-/-}\right)$. The reason for this mucus phenotype is not understood, but an optimal local ion milieu is believed to be necessary for normal mucus expansion and organisation.

The most widely used colitis model is the DSS model where rodents are given $2-5 \%$ DSS in the drinking water. $^{24}$ Inflammation typically appears after 3-5 days. When distal colon tissue sections were examined after $12 \mathrm{~h}$ exposure, the 
A

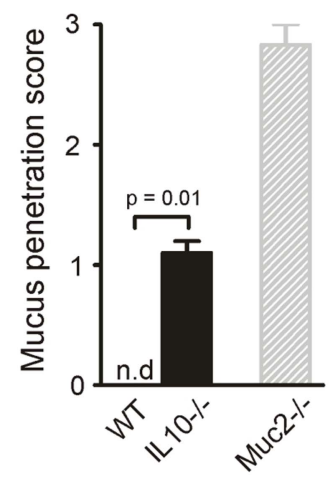

B

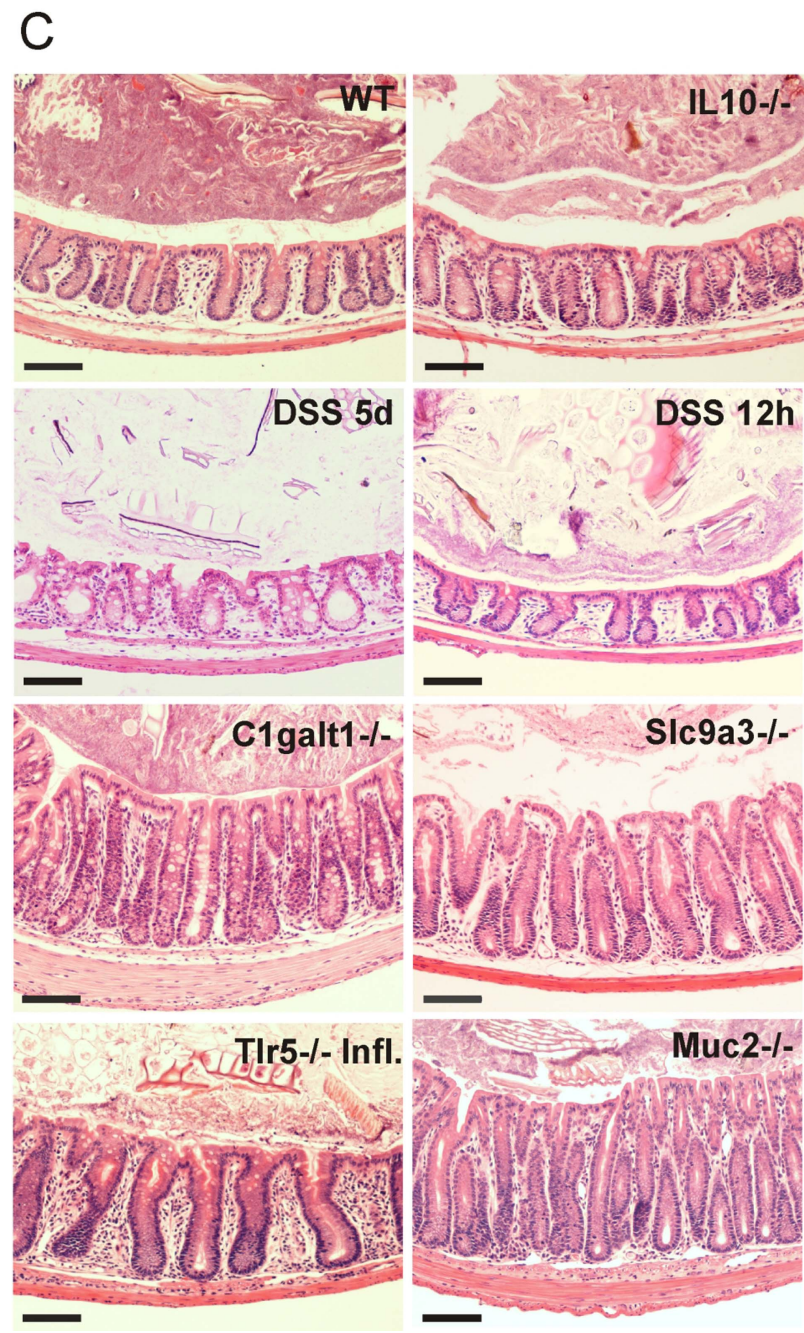

Figure 2 Bacteria penetration and inflammation in different colitis models. (A) Bacteria penetration of the inner mucus was scored for IL-1 $10^{-I-}(\mathrm{n}=5)$ and wild-type (WT; $\left.\mathrm{n}=5\right)$ mice, with Muc2 ${ }^{-l-}$, which have high numbers of bacteria in contact with the epithelium, as comparison $(n=3)$. Data are presented as mean \pm SEM. Penetration scores for all the genotypes are presented in online supplementary figure S1. nd, not detected. (B) Inflammation was monitored as

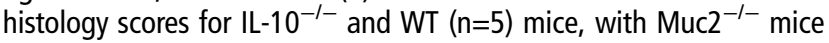
as a severely inflamed comparison $(n=3)$. Data are presented as mean ${ }_{ \pm}$SEM. Histology scores for all the genotypes are presented in online supplementary figure S1. (C) H\&E-stained tissue sections corresponding to samples in figure 1. Scale bars in all panels are $100 \mu \mathrm{m}$. DSS, $3 \%$ dextran sodium sulphate; Infl. inflamed. inner mucus layer looked relatively normal with regard to thickness and with a stratified pattern. However, bacteria had penetrated the mucus and were found close to the non-inflamed epithelium (figure 1 and online supplementary figure S1A, DSS). ${ }^{18}$ After 5 days of DSS, the inner mucus layer was lost and the tissue severely inflamed.

Bacteria recognition is part of the innate response, and a subgroup of Tlr5-deficient mice spontaneously develop colitis. ${ }^{25}$ When such animals were studied, an inner mucus layer was observed, but it was penetrated by bacteria, and massive infiltration of immune cells was observed (figures 1 and $2 \mathrm{C}$, Tlr $5^{-/}$ Infl.). The non-inflamed Tlr5-deficient mice revealed a more intact and bacteria-free inner mucus (figure 1, Tlr $5^{-/-}$ Non-Infl.).

One of the earliest knock-out animals generated was the one lacking IL-10, leading to inflammation that is dependent on the presence of bacteria. ${ }^{26} 27$ When these animals were analysed, they had very mild inflammation and normal thickness and stratified pattern of the inner mucus layer. Interestingly, their inner mucus was also penetrated by bacteria (figures 1 and $2 \mathrm{~A}$, IL-10 $\left.0^{-/}\right)$. In general terms, more severe inflammation often correlated with a high number of bacteria in contact with the epithelium. The major exception to the rule was the animals exposed to DSS for only $12 \mathrm{~h}$ where the bacteria had penetrated the inner mucus layer before development of colitis. This indicates that bacterial contact with the epithelium precedes inflammation and that bacterial contact could trigger subsequent inflammation. The IL-10 $0^{-/}$mice showed a low histology score and also a low mucus-penetration score, suggesting a relation between bacterial penetration and inflammation. Thus all tested mouse models that developed colitis showed bacteria in direct contact with the epithelium in the distal colon.

\section{The secreted mucus layers are not thinner in IL-10-deficient mice with colitis}

Because the IL- $10^{-/-}$mice have become a prototype of experimental colitis and do not have a primary defect in any mucus component, we analysed this model in more detail. As the penetration of the inner mucus layer by bacteria appears to be a hallmark of murine colitis, one could speculate that this is due to a thinner mucus layer. To address this, we measured mucus thickness in the distal colon both in vivo and ex vivo in young IL-10 $0^{-/-}$mice with low-grade inflammation and WT mice (figure $3 \mathrm{~A}, \mathrm{~B}$ ). In vivo the thickness of the total (inner and outer) mucus layer was measured directly; the loose outer mucus was then aspirated, and the thickness of the inner firmly adherent mucus was measured. The total thickness did not differ between IL-10 $0^{-/-}$and WT mice, but the adherent mucus was significantly thicker in the IL-10 $0^{-/-}$mice (figure $3 \mathrm{~A}$ ). Distal colon tissue was also mounted in a horizontal perfusion chamber, and mucus growth was followed ex vivo for $1 \mathrm{~h} .{ }^{19}$ Mucus thickness and growth during this time did not differ between IL-10 $0^{-/-}$and WT mice (figure 3B). Thus, a thinner mucus layer cannot explain why bacteria are found close to the epithelium in the IL- $10^{-/-}$mice.

The mucus stored in the goblet cells is the material used to build up and renew the secreted mucus layer. The number of goblet cells in the upper $100 \mu \mathrm{m}$ of the crypt were counted in IL-10 $0^{-/-}$and WT mouse colon sections. The amount of goblet cells did not differ between the two (figure 3C). The amount of stored mucus was assessed by measuring the area 
A

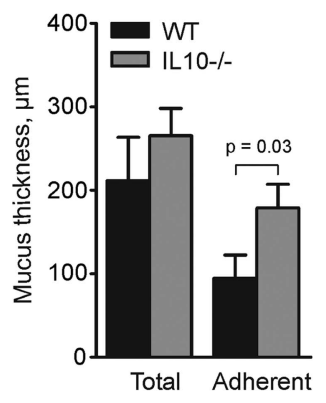

B

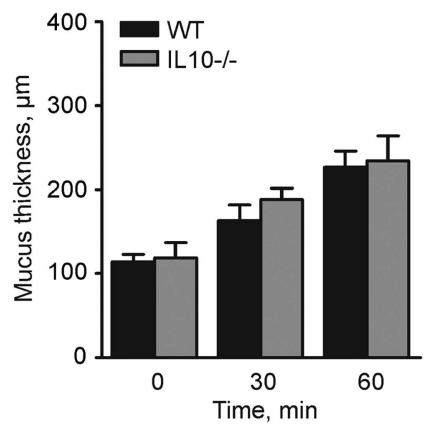

C

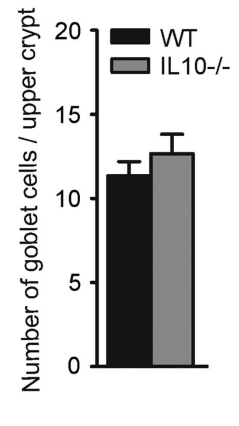

D

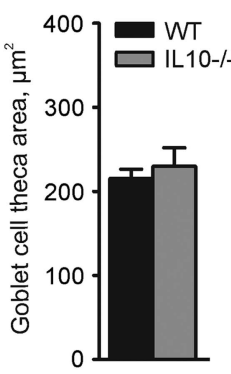

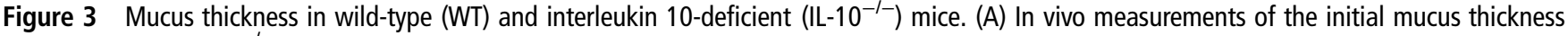
in WT $(n=7)$ and IL-10 $0^{-1-}(n=5)$ mice. The total mucus thickness (Total) was measured followed by aspiration of the mucus and measurement of the remaining mucus thickness (Adherent). (B) Ex vivo measurements of the increase in total mucus thickness over time in WT $(n=5)$ and IL-10 ${ }^{-1-}(n=6)$ mice. (C) Number of goblet cells per upper crypt in WT $(n=5)$ and IL-10 $0^{-1-}(n=5)$ mice. (D) Area of the goblet cell theca in anti-MUC2C3-stained colon section of WT $(n=5)$ and IL-10 $10^{-I-}(n=5)$ mice. Data are presented as mean \pm SEM, and the two-tailed Mann-Whitney U test was used to compare the mucus thickness in WT and IL-10 $10^{-/-}$mice.

of the mucus-filled theca in goblet cells of the upper part of the crypt. There was no significant difference in the area of the mucus-filled theca of the goblet cells between WT and IL-10 $0^{-1-}$ mice (figure $3 \mathrm{D}$ ). The stored mucus available for secretion is thus not altered in IL-10 $10^{-/}$mice.

\section{The mucus quality is defective in IL-10-deficient mice}

As the inner mucus was not found to be thinner in the IL-10 ${ }^{-/-}$ mice, we instead analysed the penetrability of the mucus to beads the size of bacteria. Colonic explants were mounted in a horizontal perfusion chamber and allowed to secrete mucus for $20 \mathrm{~min}$; fluorescent beads $(0.5$ and $2 \mu \mathrm{m})$ were then placed on top of the mucus formed. The beads were allowed to sediment for $40 \mathrm{~min}$, and the position of the beads in relation to the epithelium was determined by confocal microscopy. WT explant mucus did not allow the beads to penetrate and kept both 0.5 and $2 \mu \mathrm{m}$ beads separated from the epithelium (figure 4A). On the other hand, mucus produced by the IL- $10^{-/-}$mice was unable to create such a separation (figure 4A). To quantify mucus penetrability, we estimated the bead intensity in close proximity to the epithelium (figure $4 \mathrm{~B}$ ). In the IL-10 ${ }^{-/-}$mice, almost $50 \%$ bead intensity was found close to the epithelium compared with $1 \%$ in the WT mice. Mucus from IL-10 ${ }^{-/-}$mice was completely penetrable, while the controls had $\sim 200 \mu \mathrm{m}$ thick non-penetrable inner mucus (figure 4C). Thus, the quality of the inner mucus is compromised in IL- $10^{-/-}$mice. The thickness is based on the mucus containing beads, and as the mucus in IL- $10^{-/-}$mouse colon is very penetrable, this results in a smaller value than in figure $3 \mathrm{~B}$ where the mucus thickness is based on charcoal binding to the mucus surface.

\section{Bacteria in normal human sigmoid colon are well separated from the epithelium}

As previously described, mouse colon has a two-layer mucus system where the inner layer is devoid of bacteria, so we first asked if the same organisation was present in humans. Patients referred for colonoscopy were included after they had given informed consent. Clinical information on the control patients is compiled in online supplementary table S1. The penetrability of the secreted mucus from sigmoid biopsy samples was measured as for mouse colon, and representative Z-stack projections and corresponding normalised intensity plots for the different groups were collected (figure 5). Control patients showed an impenetrable (IP) mucus layer, on average $400 \mu \mathrm{m}$ thick, separating beads from the epithelium (figure 5A, D). To confirm that the human colonic mucus forms an inner mucus layer similar to that observed in mouse colon, ${ }^{7}$ we analysed control sigmoid samples not pretreated with laxative (figure 6C,D). Biopsy samples were fixed to preserve the mucus, immunostained for MUC2, and counterstained for DNA to visualise cell nuclei and bacteria (FISH probes do not detect all bacteria associated with the biopsy samples). Similar to the mice, a stratified mucus layer was observed with bacteria only present at the luminal surface of the mucus.

\section{Patients with active UC and a subgroup of patients with UC in remission have penetrable mucus}

Considering that increased bead penetrability correlated with bacteria close to the epithelium in mouse colitis models, we asked whether this also translated to human patients with UC. Patients with UC were divided into two groups: those in remission (Mayo endoscopic score 0) and those with active disease (Mayo endoscopic score 1-3). Clinical information on the patients with UC is compiled in table 1 . The patients with active disease displayed many more inflammatory-associated histological changes than patients in remission. The penetrability of the secreted mucus from sigmoid biopsy samples was measured, and representative Z-stack projections and corresponding normalised intensity plots for the different groups were collected (figure 5). All patients in remission (Mayo score 0) except three had a mucus thickness similar to the control patients (figure 5D). In contrast, most patients with active UC had a thinner mucus layer that was penetrable by beads. Mucus penetrability, quantified as the number of beads close to the epithelium (20 $\mu \mathrm{m}$ into the tissue and $120 \mu \mathrm{m}$ into the lumen), was $~ 40 \%$ in patients with active disease, $10 \%$ in patients in remission, and close to zero in the controls (figure 5B). The individual values for penetrability versus mucus thickness (defined as bead-containing material) show heterogeneity especially in the patients in remission (Mayo score 0) (figure 5C). Interestingly, three of the patients in remission (numbers 1, 2 and 12) produced mucus that was penetrable to the beads. When these patients with penetrable mucus $(\mathrm{P})$ were plotted separately from the remission group, the IP group looked like the control patients and the $\mathrm{P}$ group like those with active UC (Mayo score 1-3) (figure 5D). Thus, mucus secreted from 
Figure 4 Mucus penetrability in wild-type (WT; $n=5)$ and interleukin 10-deficient (IL-10-1-; $\mathrm{n}=5)$ mice. (A) Representative Z-stack projections with the respective normalised intensity plots. Scale bars $100 \mu \mathrm{m}$. (B) Percentage of the total bead intensity in close proximity to the epithelial surface $(40 \mu \mathrm{m})$. Differences between the groups were analysed using a two-tailed Mann-Whitney U test. (C) Relation between penetrable and impenetrable mucus. The black part of the bar represents impenetrable mucus and the green part mucus containing the $2 \mu \mathrm{m}$ beads (ie, penetrable).
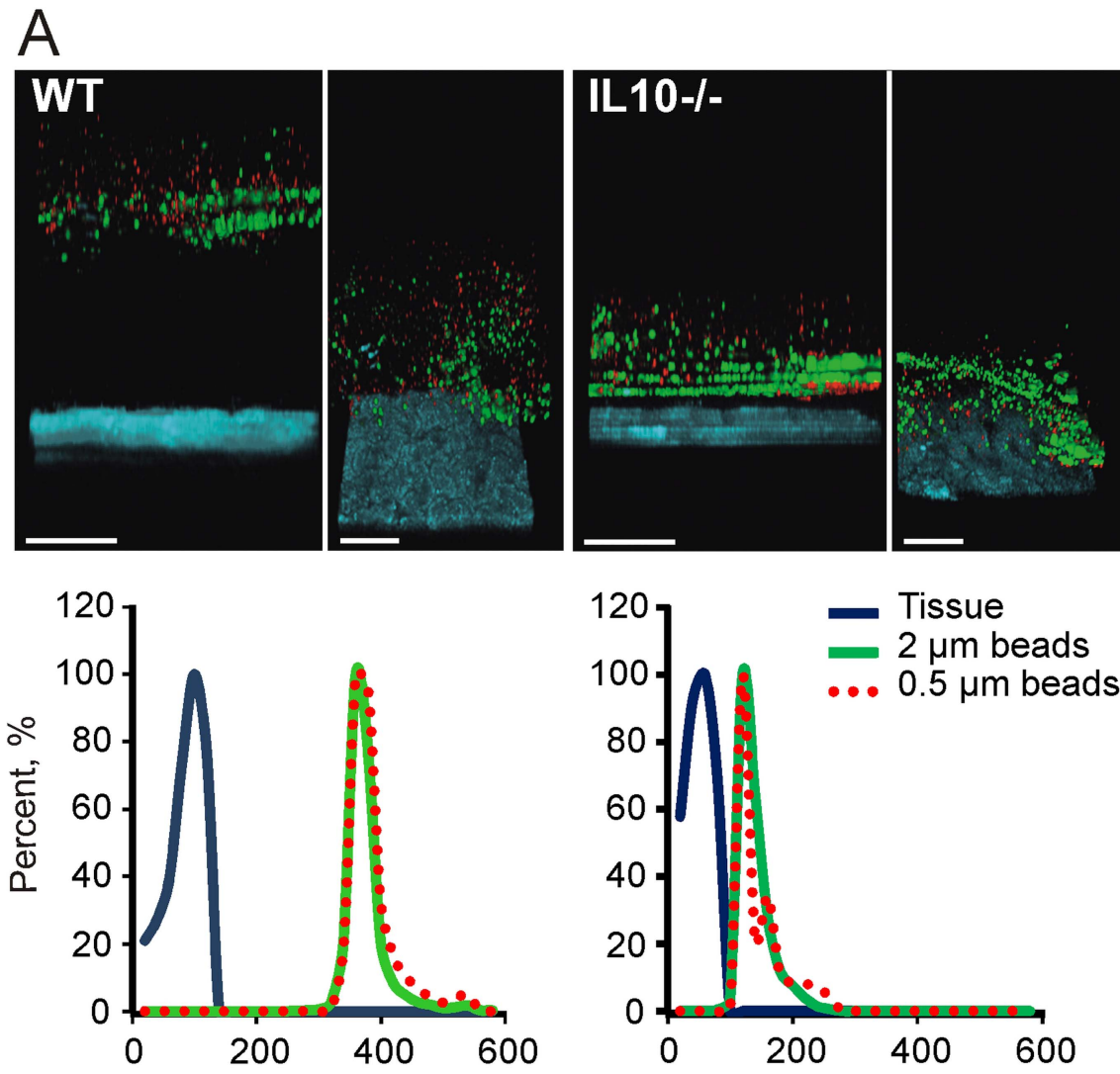

Distance, $\mu \mathrm{m}$
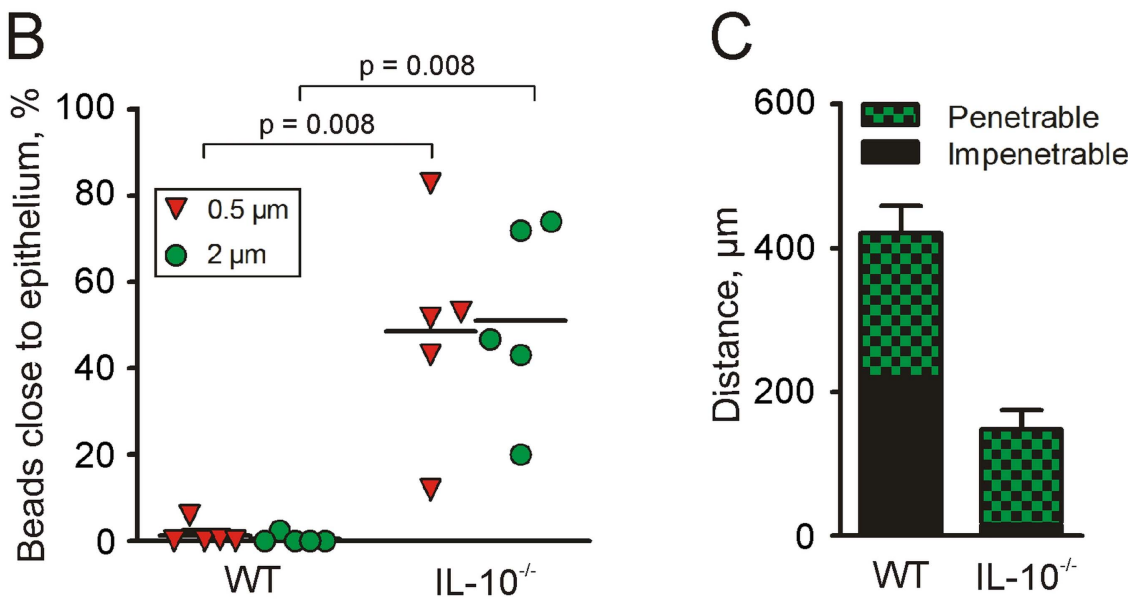

human sigmoid colon was normally dense and thick, whereas that in patients with active inflammation and in a subgroup of patients in remission was thinner and penetrable. The mucus quality did not correlate with azathioprine treatment. The number of goblet cells in the upper $150 \mu \mathrm{m}$ crypt was determined, and no differences were observed in any of the groups, indicating that patients who are either in remission or have active inflammation do not have fewer goblet cells (figure 6A). Mucus stored in goblet cells of the upper crypts measured as theca area did not differ between controls and patients in remission (figure 6B). The patients in remission who were identified to have penetrable mucus (figure $5 \mathrm{~B}, \mathrm{C}$ ) had a similar goblet cell theca area, arguing for normal amounts of stored mucus (figure 6B). The patients with active inflammation were, however, observed to have fewer filled goblet cells, seen as smaller theca areas (figure 6B), which would correspond to the thinner mucus layer observed.

We finally asked if the difference in mucus quality of the patients with active UC, which allowed beads to sediment down to the epithelium, reflected bacteria penetrating the inner mucus. Biopsy specimens taken from the same individuals as used for penetrability measurements were fixed to preserve the mucus, immunostained for MUC2, and counterstained for DNA to visualise cell nuclei and bacteria. These patients were all pretreated with oral laxative to clear the intestine for routine colonoscopy. This treatment affects the mucus and its turnover, and the fixed material shows a more voluminous and less well structured mucus in control patients also (figure 6C-b,D-b). The 
A
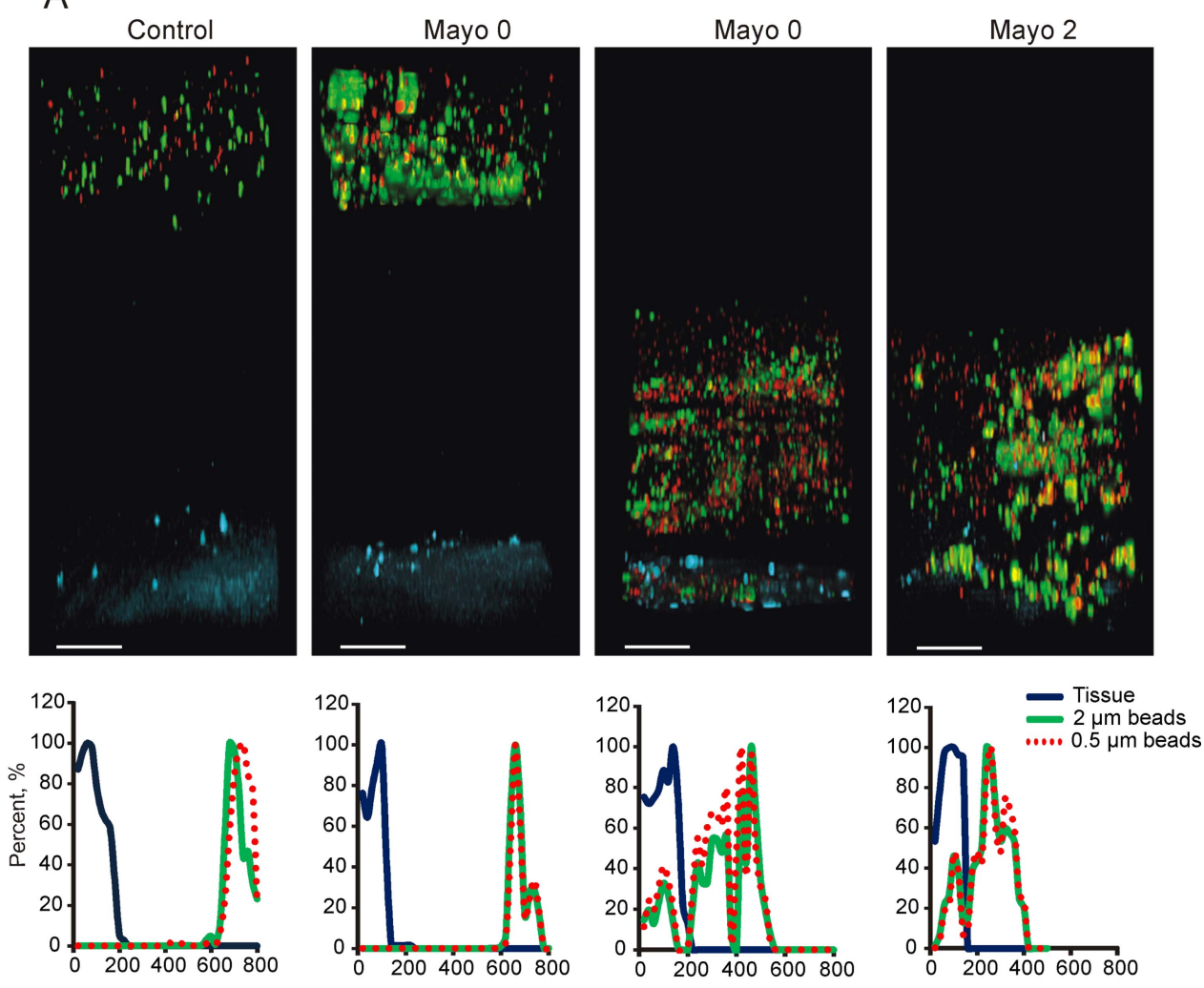

Distance, $\mu \mathrm{m}$
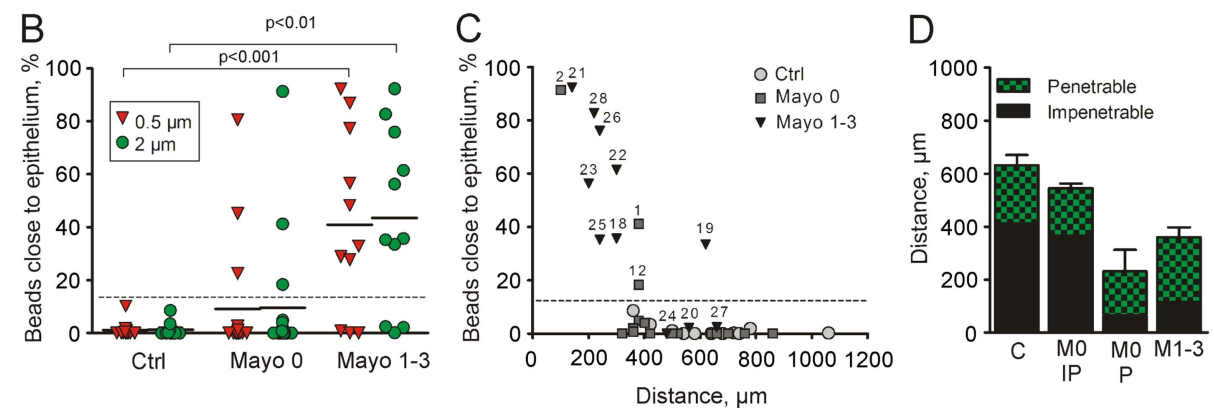

Figure 5 Mucus penetrability in human colonic biopsy samples from controls $(n=12)$ and patients with ulcerative colitis (Mayo $0, n=17$, Mayo $1-3, n=11$ ). (A) Representative Z-stack projections with the respective normalised intensity plots. Scale bars $100 \mu \mathrm{m}$. (B) Percentage of total bead intensity in close proximity to the epithelial surface $(<120 \mu \mathrm{m})$. Differences between groups were analysed using the Kruskal-Wallis test with Dunns' correction for multiple comparisons (primary analysis $p=0.0009$ for the $2 \mu \mathrm{m}$ beads and $p=0.0004$ for the $0.5 \mu \mathrm{m}$ beads). (C) Percentage of beads in relation to total mucus thickness of individual patients. Numbers refer to patient numbers given in table 1. (D) Relation between penetrable and impenetrable mucus of the $2 \mu \mathrm{m}$ green beads. C, control; M0 IP, patients with Mayo endoscopic score 0 and impenetrable mucus; M0 P, Mayo score 0 and penetrable mucus (patients number 1, 2 and 12); M1-3, Mayo score 1-3.

amount of mucus that remained attached to the epithelial surface varied between the biopsy specimens, which excluded evaluation of all patients. In comparison with the laxativetreated control patients, the patients with inflammation had less attached mucus, and bacteria penetrated this mucus and reached the epithelium (figure 6C-c, D-c).

\section{DISCUSSION}

As demonstrated previously, ${ }^{7}$ the inner mucus layer of normal healthy colon of mice forms a barrier that separates bacteria from the epithelium. This was shown by Carnoy fixation of intact tissue without previous washing of the luminal content. We have now studied a number of different colitis models and confirmed mucus defects in all of them. It is well established that intestinal bacteria are necessary for all these models and that the composition of the microbiota influences the severity and outcome of the inflammation. Muc2-null mice totally lack mucus and spontaneously develop severe inflammation, ${ }^{7} 15$ while other models all have a more or less intact inner mucus layer which is, however, penetrated by bacteria. The glycan-deficient Core 1-deficient mice have less well glycosylated Muc2 mucin, which is probably easier to degrade by the bacteria. $^{21}$ DSS induces inflammation initiated by a direct toxic effect on the mucus. ${ }^{18}{ }^{28} \mathrm{Tlr} 5^{-/-}$mice have a thinner and defective inner mucus layer. ${ }^{29}$ Nhe3-deficient and IL-10 $0^{-/-}$ mice display a morphologically stratified inner mucus layer that is still penetrable to bacteria. ${ }^{22}{ }^{23}$ These data argue for an impaired mucus layer that allows bacteria to penetrate as a potential mechanism involved in the development of colon inflammation. 


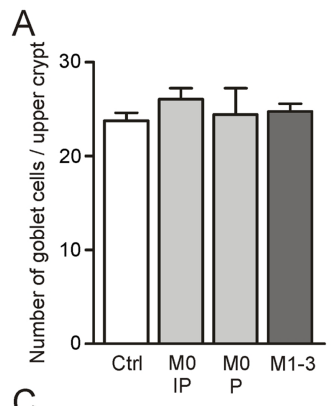

B
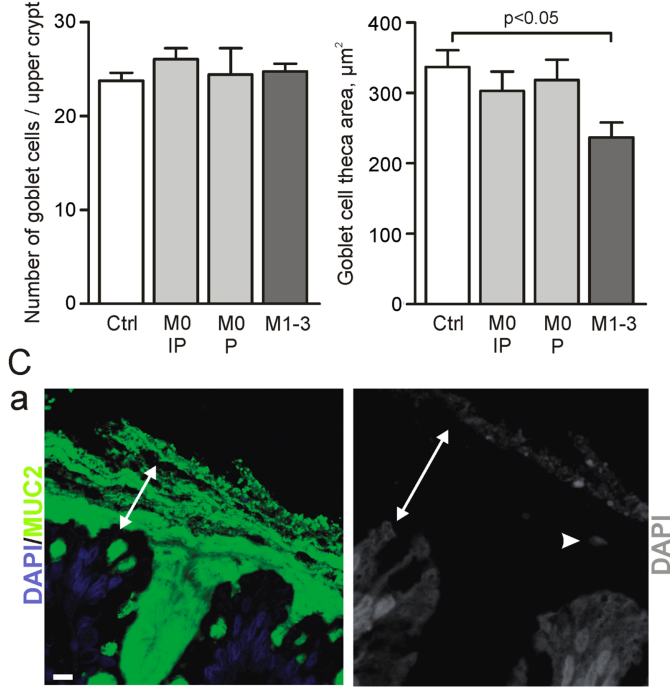

b
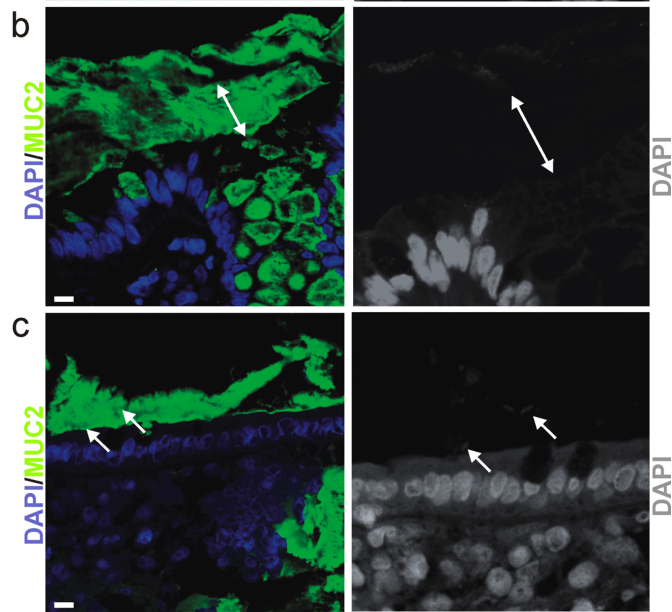

D $\underline{a}$

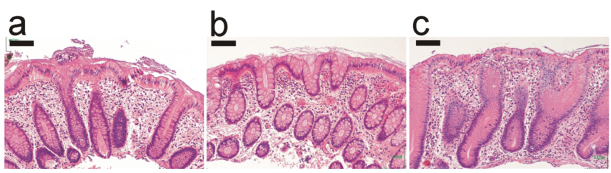

Figure 6 Mucus-filled goblet cells and bacteria in fixed human sigmoid biopsy samples. Human colon biopsy samples were Carnoy fixed to preserve the mucus and immunostained for MUC2 in combination with 4',6-diamidino-2-phenylindole (DAPI) for DNA in nuclei and bacteria. (A) The number of goblet cells per upper crypt was determined in fixed and MU2-stained sections from patients with ulcerative colitis (UC) and controls. (B) The goblet cell theca area was measured in fixed and MU2-stained sections from patients with UC and controls. Data are presented as mean \pm SEM, and the Kruskal Wallis test with Dunn's correction for multiple comparisons was used to compare the UC patients with the control group. Ctrl, control; MO IP, patients with endoscopic Mayo score 0 and impenetrable mucus; M0 P, Mayo score 0 and penetrable mucus (patients number 1, 2 and 12); M1-3, Mayo score 1-3. (C) Sections from human sigmoid biopsy samples stained for MUC2 (green) and DAPI (blue). (a) A biopsy specimen collected and directly fixed from sigmoid colon of a control patient without preceding laxative treatment. (b) A biopsy specimen from a control patient included in the penetrability study who was pretreated with laxatives before colonoscopy. (c) Biopsy specimen from patient with UC pretreated with laxative and with a Mayo endoscopic score of 2 at colonoscopy. Pictures to the right only show the DAPI staining. Bacteria (arrows) are found on the outer surface of the mucus in control patients ( $a$ and $b$ ). Bacteria are found inside the inner mucus and close to the epithelium in the patient with active UC (c). Some detached cells can be observed (arrowhead). (D) H\&E-stained tissue each corresponding to parts $a-c$ in (C). Scale bars are $10 \mu \mathrm{m}(C)$ and $100 \mu \mathrm{m}$ (D).
Assuming that the inner mucus layer of the colon is vitally important for colon barrier function, a thinner mucus layer could be associated with inflammation. However, mucus thickness alone was not a useful indicator of mucus barrier function since, for example, the IL-10-deficient mice had a thicker inner attached mucus layer. Instead, these animals showed increased mucus secretion, maybe in an attempt to overcome the mucus barrier defect.

We can now show that the sigmoid colon of healthy humans also has an inner stratified MUC2-containing mucus layer that separates bacteria from the epithelium. Bacterial contact with the epithelium has previously been observed in inflamed intestinal samples, but it was only analysed on fixed biopsy samples. ${ }^{16} 17$ The mucus is then often lost or degraded, problems that can hamper the evaluation. We have now studied the protective quality of the mucus by assessing its ability to restrict small spherical particles from sedimenting down to the epithelial surface to illustrate its property of excluding bacteria close to the epithelium. This was performed on live explant under physiological conditions to gain information about its normal in vivo function. The distance between beads and cells was $\sim 200 \mu \mathrm{m}$ in the mouse, something that should be compared with $\sim 50 \mu \mathrm{m}$ thick inner mucus layer as measured after aspiration of the outer loose mucus layer. ${ }^{7}$ That the beads did not pass all the way down to $50 \mu \mathrm{m}$ suggests that the outer mucus layer is not homogeneous and that it is denser close to the border between the inner and outer mucus layer. This is in line with the prediction of proteolytic activity being responsible for the conversion from inner dense to outer loose mucus layer. ${ }^{7}$ The mucus separating the beads was $\sim 400 \mu \mathrm{m}$ thick in humans, and thus the inner mucus layer can be predicted to be about twice as thick in humans as in mice.

The IL- $10^{-/-}$model is one of the oldest colitis models studied, in which the inflammation can be explained by the loss of the anti-inflammatory effects of IL-10. ${ }^{30}$ The proinflammatory initiating effect is mediated by colonic mononuclear phagocytes of the innate defence system. ${ }^{31}$ The IL- $10^{-/-}$mice kept in our animal house display only minor histological inflammatory signs, but still have a mucus layer that is penetrable to both beads and bacteria. This argues for a link between mucus properties and the immune system and cytokines produced. The mechanism behind the IL-10 effect on the mucus-producing goblet cells is not understood, but the number of goblet cells and size of the theca with the mucus granulae in the goblet cells do not differ from WT mice. This implies changes not related to mucus amount and may suggest other mechanisms. Mucus organisation and expansion at secretion depend on the external milieu, and alterations could have devastating effects. ${ }^{13}{ }^{32}$ Mucus organisation is also a result of additional cross-linking and processing, defects in which could also contribute to the identified penetrable mucus.

UC is not caused by pathogenic bacteria, but is rather the result of abnormal reactions to the normal microbiota possibly associated with dysbiosis. ${ }^{3} 433 \quad 34$ Antibiotic treatments and faecal deviation can sometimes be beneficial for patients with colitis, ${ }^{35} 36$ although the best arguments for the importance of lowering the load of commensal bacteria in contact with the epithelium are based on mouse studies. The surface epithelial cell layer can probably withstand and handle some bacteria, but will probably have difficulty withstanding substantial direct bacterial contact for a long period. Extensive bacterial contact will cause bacterial leakage into the tissue, something that could trigger the subepithelial adaptive immune system.

Most of the patients with UC in remission (Mayo score 0) had, like the control patients, a thick inner mucus layer that was 
not penetrated by fluorescent beads. However, three of these patients had mucus that was more or less totally penetrable by the beads, but did not have smaller goblet cell theca area in analogy with the IL- $10^{-/-}$mice. Two of these patients had primary sclerosing cholangitis. As all the spontaneous mouse colitis models had mucus that was penetrable to bacteria, it can be expected that some patients with UC might also have mucus defects making their mucus less protective. The reason for the observed mucus alterations in patients with active colitis may be higher mucus secretion as shown by the same number of, but less well filled, goblet cells-observed as smaller theca area or more empty goblet cells-described after PAS staining as goblet cell depletion. This characteristic is mainly attributable to more severely inflamed tissue and not observed in inactive disease or the less inflamed IL- $10^{-/}$animals. As generation of the enormously large MUC2 polymeric network is time consuming and difficult, a high demand and fast turnover, as in inflammation, are likely to generate mucus of poorer quality. ${ }^{37}$ This was recently illustrated by studies of colon ischaemia where reperfusion emptied the crypt goblet cells with a concomitant clearance of bacteria. ${ }^{38}$ After this, the goblet cells were not refilled for many hours, and another challenge would be less efficiently handled. An inner mucus gel that is less dense with MUC2 mucin is likely to be more penetrable, but other more sophisticated mechanisms are likely to be involved. The genetic and environmental factors causing and maintaining UC are probably numerous. Our observations suggest that the inner mucus and its barrier function is an important factor in limiting bacterial contact with the epithelium and that defects in this system might trigger inflammation. The properties of colon mucus are also shown to be dynamic and probably modulated by both bacteria and host factors using only partly known mechanisms. Understanding these mechanisms may provide novel approaches for prolonging remission periods for patients with UC.

Acknowledgements Drs Herbert Helander and Mats Wolving are acknowledged for pathological examinations.

Contributors MEVJ and GCH conceived the original idea. MEVJ, JKG, JH-L, HS, $\mathrm{KSJ}$ and GCH designed the study, analysed the data and wrote the manuscript. MEVJ, JKG and JH-L conducted the experiments, and analysed the data. LX, HX, FKG, FAC and ATG provided genetically modified mouse tissues. KSJ and HS analysed the patients.

Funding This work was supported by the Swedish Research Council (No 7461, 21027), The Swedish Cancer Foundation, The Knut and Alice Wallenberg Foundation, IngaBritt and Arne Lundberg Foundation, Sahlgren's University Hospital (LUA-ALF), Wilhelm and Martina Lundgren's Foundation, Torsten och Ragnar Söderbergs Stiftelser, The Sahlgrenska Academy, National Institutes of Health (U01AI095473, R01DK073638, the content is solely the responsibility of the authors and does not necessarily reflect the the National Institutes of Health), The Swedish Foundation for Strategic Research-The Mucus-Bacteria-Colitis Center (MBC) of the Innate Immunity Program and the Assar Gabrielsson Foundation.

\section{Competing interests None.}

Ethics approval All animal studies were approved by the animal ethics committee of the respective universities: Gothenburg, Emroy, Arizona and Oklahoma. The human studies were approved by the Human Research Ethics Committee, University of Gothenburg.

\section{Patient consent Obtained}

Provenance and peer review Not commissioned; externally peer reviewed.

Open Access This is an Open Access article distributed in accordance with the Creative Commons Attribution Non Commercial (CC BY-NC 3.0) license, which permits others to distribute, remix, adapt, build upon this work non-commercially, and license their derivative works on different terms, provided the original work is properly cited and the use is non-commercial. See: http://creativecommons.org/licenses/by-n/3.0/

\section{REFERENCES}

1 Xavier RJ, Podolsky DK. Unravelling the pathogenesis of inflammatory bowel disease. Nature 2007:448:427-34.
2 Sartor RB. Therapeutic manipulation of the enteric microflora in inflammatory bowel diseases: antibiotics, probiotics, and prebiotics. Gastroenterology 2004:126:1620-33.

3 Khor B, Gardet A, Xavier RJ. Genetics and pathogenesis of inflammatory bowel disease. Nature 2011:474:307-17.

4 Danese S, Fiocchi C. Ulcerative Colitis. New Engl J Med 2011;365:1713-25.

5 Schirbel A, Fiocchi C. Inflammatory bowel disease: established and evolving considerations on its etiopathogenesis and therapy. J Dig Dis 2010;11:266-76.

6 Johansson MEV, Hansson GC. Keeping Bacteria at a Distance. Science 2011;334:182-3

7 Johansson MEV, Phillipson M, Petersson J, et al. The inner of the two Muc2 mucin dependent mucus layers in colon is devoid of bacteria. Proc Natl Acad Sci USA 2008; 105:15064-9.

8 van der Waaij LA, Harmsen HJM, Madjipour M, et al. Bacterial population analysis of human colon and terminal ileum biopsies with $16 \mathrm{~S}$ rRNA-based fluorescent probes: commensal bacteria live in suspension and have no direct contact with epithelial cells. Inflamm Bowel Dis 2005;11:865-71.

9 Swidsinski A, Loening-Baucke $V$, Lochs $H$, et al. Spatial organization of bacterial flora in normal and inflamed intestine: a fluorescence in situ hybridization study in mice. World J Gastroenterol 2005:11:1131-40.

10 Matsuo K, Ota $H$, Akamatsu T, et al. Histochemistry of the surface mucous gel layer of the human colon. Gut 1997;40:782-9.

11 Johansson MEV, Larsson JM Holmen, Hansson GC. The two mucus layers of colon are organized by the MUC2 mucin, whereas the outer layer is a legislator of host-microbial interactions. Proc Natl Acad Sci USA 2011;108:4659-65.

12 Godl K, Johansson MEV, Karlsson H, et al. The N-termini of the MUC2 mucin form trimers that are held together within a trypsin-resistant core fragment. J Biol Chem 2002;277:47248-56.

13 Ambort D, Johansson MEV, Gustafsson JK, et al. Calcium and pH-dependent packing and release of the Gel-forming MUC2 Mucin. Proc Natl Acad Sci USA 2012; 109:5645-50.

14 Johansson MEV, Thomsson KA, Hansson GC. Proteomic analyses of the two mucus layers of the colon barrier reveal that their main component, the Muc2 mucin, is strongly bound to the FCGBP protein. J Proteome Res 2009;8:3549-57.

15 Van der SM, de Koning BA, De Bruijn AC, et al. Muc2-deficient mice spontaneously develop colitis, indicating that MUC2 is critical for colonic protection. Gastroenterology 2006;131:117-29.

16 Swidsinski A, Loening-Baucke V, Theissig F, et al. Comparative study of the intestinal mucus barrier in normal and inflamed colon. Gut 2007;56:343-50.

17 Schultsz C, van den Berg FM, en Kate FW, et al. The intestinal mucus layer from patients with inflammatory bowel disease harbors high numbers of bacteria compared with controls. Gastroenterology 1999;117:1089-97.

18 Johansson MEV, Gustafsson JK, Sjoberg KE, et al. Bacteria penetrate the inner mucus layer before inflammation in the Dextran sulfate colitis model. PLOS ONE 2010;5:e12238

19 Gustafsson JK, Ermund A, Johansson MEV, et al. An ex vivo method for studying mucus formation, properties and thickness in human colonic biopsies and mouse small and large intestinal explants. Amer J Physiol Gastrointest Liver Physiol 2011:302:G430-8.

20 Lewis JD, Chuai S, Nessel L, et al. Use of the noninvasive components of the Mayo score to assess clinical response in ulcerative colitis. Inflamm Bowel Dis 2008;14:1660-6

21 Fu J, Wei B, Wen T, et al. Loss of intestinal core 1-derived 0 -glycans causes spontaneous colitis. J Clin Invest 2011;121:1657-66.

22 Schultheis PJ, Clarke LL, Meneton $\mathrm{P}$, et al. Renal and intestinal absorptive defects in mice lacking the NHE3 Na+/H+ exchanger. Nat Genet 1998;19:282-5.

23 Laubitz D, Larmonier CB, Bai A, et al. Colonic gene expression profile in NHE3-deficient mice: evidence for spontaneous distal colitis. Am J Physiol Gastrointest Liver Physiol 2008;295:G63-77.

24 Isao 0 , Shigeru $H$, Masahiro $Y$, et al. A novel method in the induction of reliable experimental acute and chronic ulcerative colitis in mice. Gastroenterology 1990;98:694-702.

25 Vijay-Kumar M, Sanders CJ, Taylor RT, et al. Deletion of TLR5 results in spontaneous colitis in mice. J Clin Invest 2007;117:3909-21.

26 Kuhn R, Lohler J, Rennick D, et al. Interleukin-10-deficient mice develop chronic enterocolitis. Cell 1993;75:263-74.

27 Sellon RK, Tonkonogy S, Schultz M, et al. Resident enteric bacteria are necessary fo development of spontaneous colitis and immune system activation in interleukin-10-deficient mice. Infect Immun 1998:66:5224-31.

28 Yan Y, Kolachala V, Dalmasso G, et al. Temporal and Spatial Analysis of Clinica and Molecular Parameters in Dextran Sodium Sulfate Induced Colitis. PLOS ONE 2009;4:e6073.

29 Carvalho FA, Koren 0, Goodrich JK, et al. Transient inability to manage proteobacteria promotes chronic gut inflammation in TLR5-deficient mice. Cell Host Microbe 2012:12:139-52.

30 Izcue A, Coombes JL, Powrie F. Regulatory Lymphocytes and Intestinal Inflammation. Annu Rev Immunol 2009:27:313-38. 
31 Hoshi N, Schenten D, Nish SA, et al. MyD88 signalling in colonic mononuclear phagocytes drives colitis in IL-10-deficient mice. Nat Commun 2012;3:1120

32 Gustafsson JK, Ermund A, Ambort D, et al. Bicarbonate and functional CFTR channel is required for proper mucin secretion and link Cystic Fibrosis with its mucus phenotype. J Exp Med 2012;209:1263-72.

33 Mannon PJ, Hornung RL, Yang Z, et al. Suppression of inflammation in ulcerative colitis by interferon-beta-1a is accompanied by inhibition of IL-13 production. Gut 2011;60:449-55.

34 Fiocchi C. Inflammatory bowel disease: etiology and pathogenesis. Gastroenterology 1998;115:182-205.
35 Gilat T, Leichtman G, Delpre G, et al. A comparison of metronidazole and sulfasalazine in the maintenance of remission in patients with ulcerative colitis. J Clin Gastroenterol 1989:11:392-5.

36 Gionchetti P, Rizzello F, Ferrieri A, et al. Rifaximin in patients with moderate or severe ulcerative colitis refractory to steroid-treatment: a double-blind, placebo-controlled trial. Dig Dis Sci 1999;44:1220-1.

37 Johansson MEV, Ambort D, Pelaseyed T, et al. Composition and functional role of the mucus layers in the intestine. Cell Mol Life Sci 2011;68:3535-641.

38 Grootjans J, Hundscheild IH, Lenaerts $\mathrm{K}$, et al. Ischemia-induced mucus barrier loss and bacterial penetration are rapidly counteracted by increased goblet cell secretory activity in human and rat colon. Gut 2012;62:250-8. 